\title{
Cisto nasolabial bilateral: relato de dois casos e revisão daliteratura
}

\section{Nasolabial bilateral cyst two case report and a review of the literature}

\author{
José A. de P. Felix ${ }^{1}$, Paulo J. F. Ferreira ${ }^{2}$, \\ Rodrigo Correa ${ }^{3}$, Rodrigo Cantini ${ }^{4}$, \\ Rosalvo M. Neto ${ }^{5}$, Felippe Felix ${ }^{6}$
}

\author{
Palavras-chaves: cisto não-odontogênico, \\ cisto nasolabial.
}

Key words: nonodontogenic cyst, nasolabial cyst.

\section{Resumo / Summary}

cisto nasolabial é um raro cisto da linha média facial. Sua ocorrência bilateral é extremamente incomum. Poucos casos são descritos na literatura. A manifestação clínica representa o mais importante critério de diagnóstico. A terapia consiste em remoção cirúrgica intraoral. Neste artigo, os autores apresentam dois casos de cisto nasolabial bilateral, ambos em pacientes de meia idade do sexo feminino, e uma revisão da literatura sobre o tema.
T

he nasolabial cyst is an uncommon midfacial lesion. Its bilateral occurrence is extremely rare. Few cases ara described in the literature. This clinical manifestation represents the most important criterion for diagnosis. Therapy consists of surgical removal using an intraoral approach. The authors present two cases of bilateral nasolabial cysts, both in middle aged females, and a review of the literature is included.

\footnotetext{
1 Prof. Adjunto da Disciplina de Otorrinolaringologia da Faculdade de Medicina da Universidade Federal Fluminense.

2 Prof. Assistente da Disciplina de O torrinolaringologia da Faculdade de Medicina da Universidade Federal Fluminense.

3 Pós Graduando da Disciplina de Otorrinolaringologia da Faculdade de Medicina da Universidade Federal Fluminense.

4 Pós-Graduando da Disciplina de O torrinolaringologia da Faculdade de Medicina da Universidade Federal Fluminense.

${ }^{5}$ Pós Graduando da Disciplina de Otorrinolaringologia da Faculdade de Medicina da Universidade Federal Fluminense. ${ }^{6}$ Acadêmico de Medicina do $6^{\circ}$ ano da Universidade Federal Fluminense.

Endereço para correspondência: José Antonio de Paula Felix - Rua Cel. Moreira César 229, sala 1815 24230-053 Icaraí, Niterói RJ Tel/fax: (0xx21) 2710-6220 - E-mail: felfelix@cruiser.com.br 


\section{INTRODUÇÃO}

Os cistos nasolabiais foram originalmente mencionados por Zuckerkandl em 1882. É um raro cisto embrionário, não odontogênico, com origem na região do lábio superior logo abaixo da asa do nariz. Possui ampla denominação como: cisto nasoalveolar, cisto do vestíbulo nasal, cisto mucóide do nariz, mas o nome cisto nasolabial, criado por Rao em 1955, é atualmente considerado o mais adequado ${ }^{1}$.

A ocorrência deste tipo de lesão é incomum sendo a apresentação bilateral extremamente rara². De acordo com a literatura mundial, apenas $10 \%$ dos casos tem ocorrência bilateral $^{3}$. Os primeiros autores a apresentarem casos de cistos nasolabiais bilaterais foram Kofler em 1916 e Terracol em $1936^{4}$.

O propósito deste trabalho é apresentar dois casos de pacientes com cisto nasolabial bilateral, um diagnosticado concomitantemente e outro em épocas diferentes, além de promover uma revisão da literatura sobre o tema.

\section{APRESENTAÇÃO DOCASO 1}

Paciente do sexo feminino, 43 anos de idade, cor parda, natural do Ceará, apresentou-se com queixa de aumento de tamanho e dor próximo a fossa nasal direita acompanhado de obstrução nasal em julho de 1998. Relata, ainda, que 0 inchaço foi percebido há aproximadamente 1 ano e que progressivamente aumentava de tamanho.

Ao exame físico otorrinolaringológico foi notado aumento doloroso de consistência dura, flutuante com coloração normal medindo aproximadamente $2,5 \mathrm{~cm}$ acompanhado de elevação da asa nasal direita e apagamento do sulco nasolabial correspondente (Figura 1). Foi solicitado radiografia simples dos seios paranasais não sendo possível identificar a lesão. Foi feito diagnóstico clínico topográfico de cisto nasolabial e indicado tratamento cirúrgico. É importante ressaltar que o lado esquerdo encontrava-se sem alteração.

Realizado exerése cirúrgica sob anestesia geral em setembro de 1998 por via intra-oral. Feito incisão no sulco gengivolabial entre 0 incisivo central e o primeiro molar superior direito com retirada da lesão com dissecção romba. 0 cisto possuía aproximadamente $2,5 \mathrm{~cm}$ com secreção amarelada em seu interior. Exame histológico revelou parede cística fibrosa com a luz revestida por epitélio pseudoestratificado cilíndrico envolvendo células mucossecretoras, caliciformes; em duas áreas o epitélio escamoso metaplásico levanta o epitélio cilíndrico levando-o a atrofia (Figura 2). Acompanhamento de 5 meses pós-operatório assintomático.

Em março de 2000, retornou relatando que, a partir de dezembro de 1999, percebeu que havia um crescimento semelhante ao anterior no lado esquerdo. No exame otorrinolaringológico encontramos tumefação cística de aproximadamente $2 \mathrm{~cm}$ no sulco nasolabial esquerdo (Figura

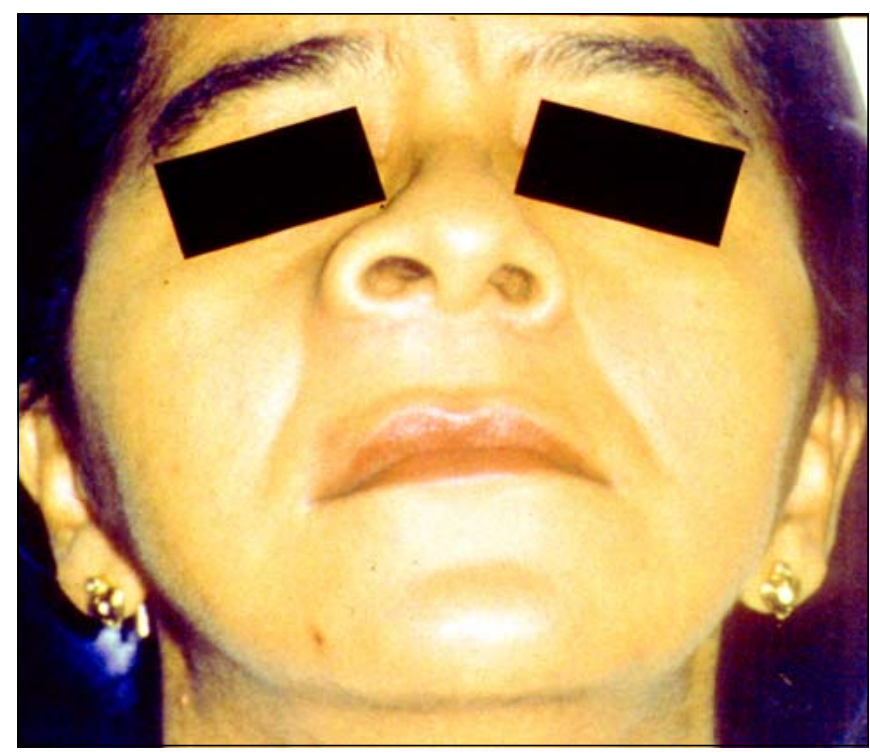

Figura 1. Tumoração na região da asa nasal direita.

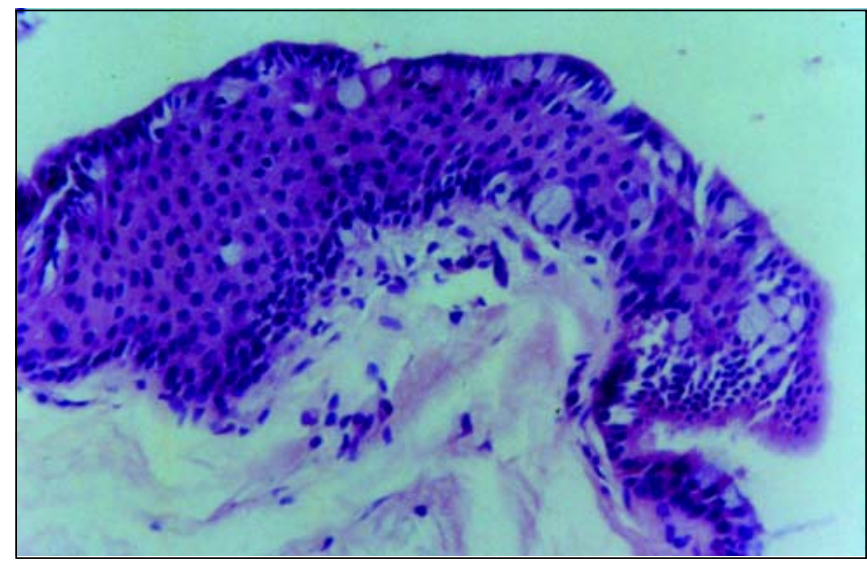

Figura 2. Área de metaplasia escamosa do epitélio que reveste a luz do cisto, identificando-se ainda algumas células caliciformes (H. E. 200x).

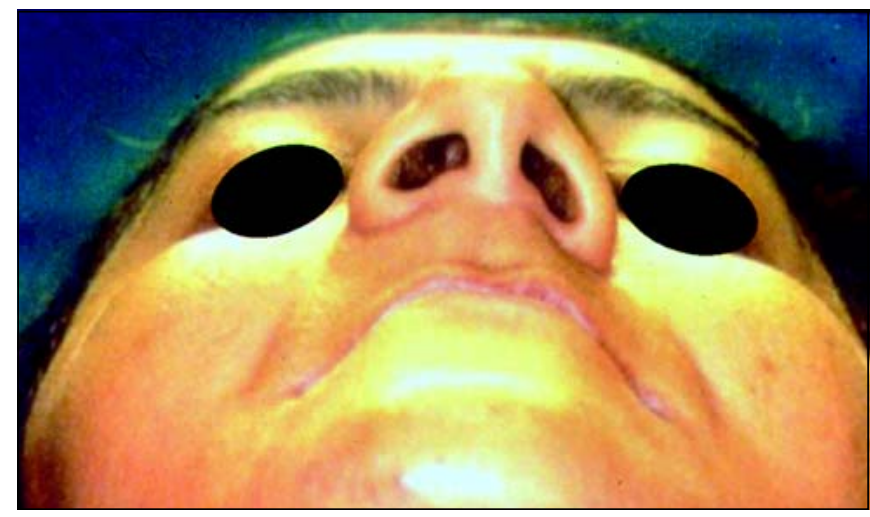

Figura 3. Tumoração da região da asa nasal esquerda. 


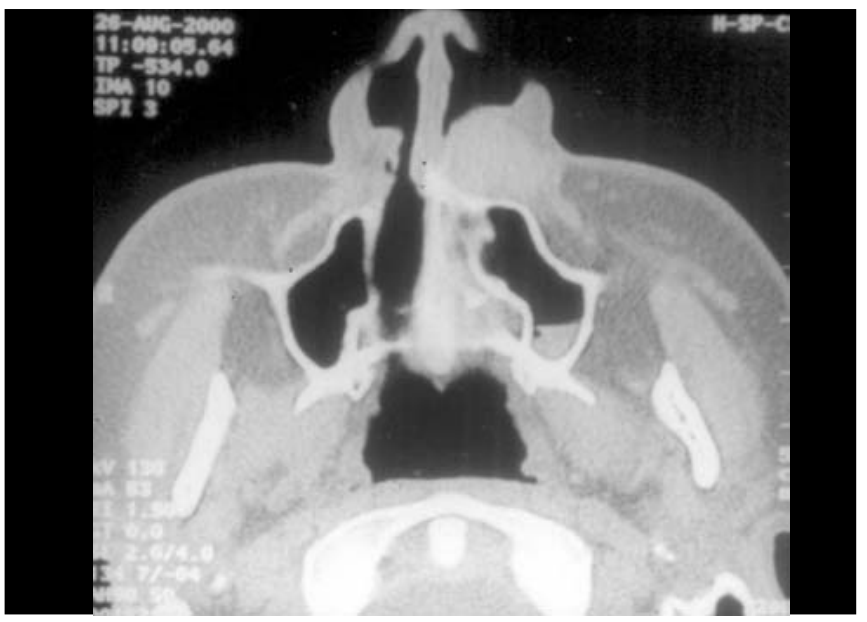

Figura 4. Tomografia Computadorizada dos seios paranasais evidenciando tumoração arredondada em vestíbulo nasal esquerdo restrita a partes moles da região pré maxilar.

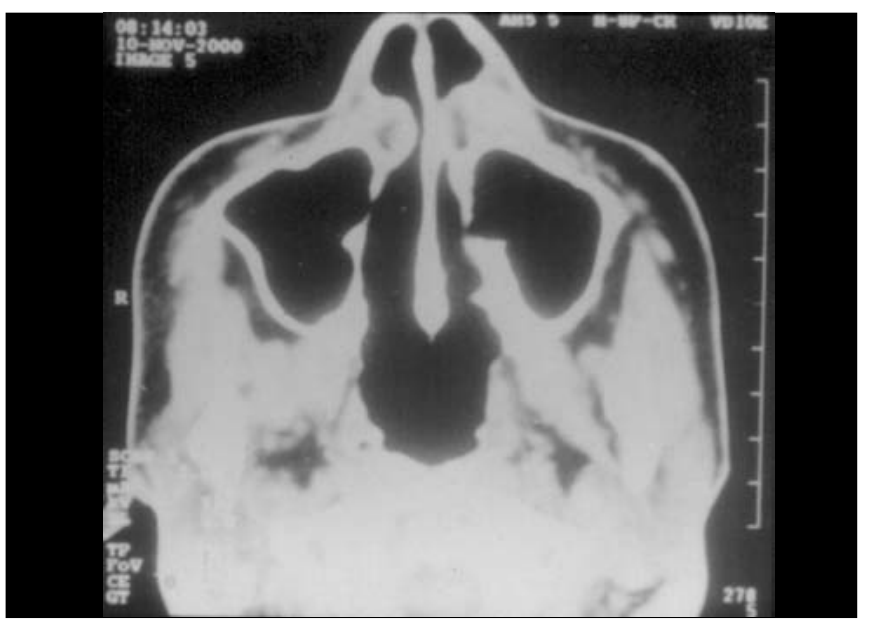

Figura 6. Tomografia Computadorizada dos seios paranasais evidenciando material com densidade de partes moles preenchendo o assoalho de ambas fossas nasais.

3). Solicitada tomografia computadorizada dos seios paranasais que evidenciou uma tumoração arredondada em vestíbulo nasal esquerdo restrita a partes moles da região pré-maxilar (Figura 4).

A paciente foi submetida a exerése cirúrgica do novo cisto em setembro de 2000. Exame patológico mostrou aspecto semelhante ao anterior. Houve boa evolução no acompanhamento pós-operatório por 6 meses.

\section{APRESENTAÇÃODOCASO 2}

Paciente do sexo feminino, 31 anos de idade, raça negra, natural e moradora de Guapimirim (RJ) apresentouse com queixa principal de "caroço no nariz". Refere aparecimento de tumoração em assoalho de fossa nasal esquerda há 2 anos com aumento progressivo de tamanho, passando a abaular também o assoalho da fossa nasal direita (Figura 5). Negava dor, epistaxe, rinorréia ou obstrução

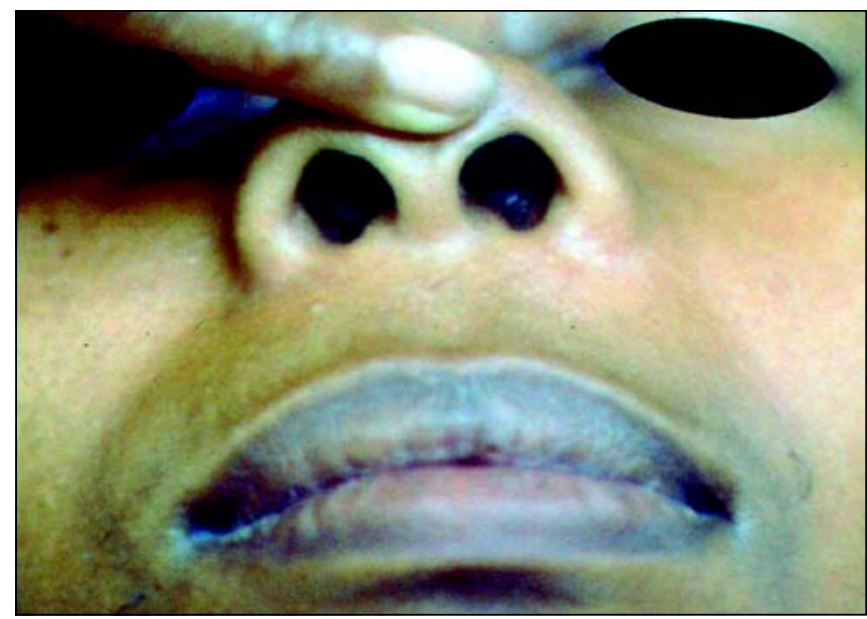

Figura 5. Tumorações em região de asa nasal direita e esquerda.

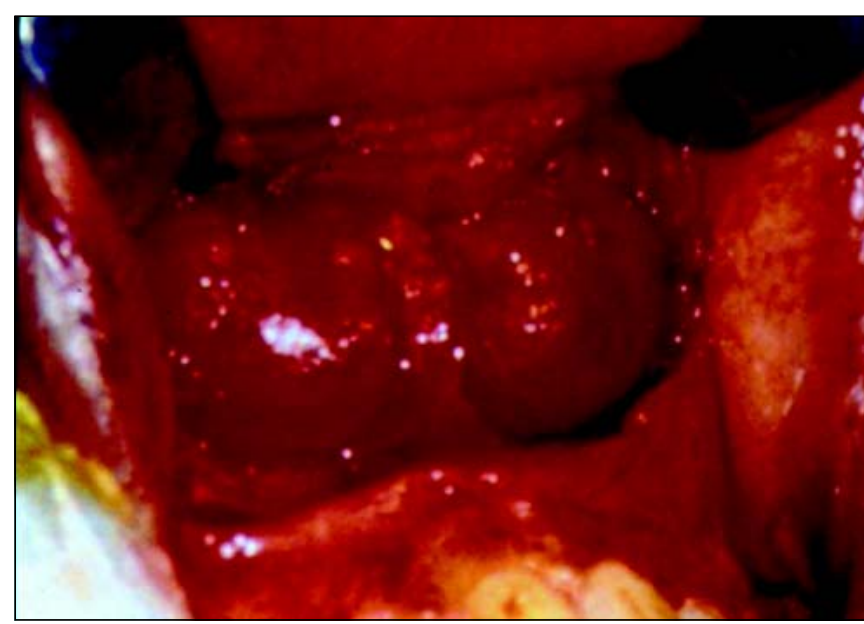

Figura 7. Exposição cirúrgica de ambos o cistos nasolabiais.

nasal. Ao exame físico otorrinolaringológico apresentava abaulamento em ambas fossas nasais, sendo de maior tamanho no lado esquerdo, sem sinais flogistícos, ocupando aproximadamente $30 \%$ da fossa nasal esquerda e $20 \%$ da fossa nasal direita; além disso havia apagamento do sulco nasolabial bilateral.

Solicitada tomografia computadorizada dos seios paranasais que evidenciou material com densidade de partes moles preenchendo o assoalho de ambas fossas nasais, organizado de forma arredondada (Figura 6). A endoscopia nasal confirmou abaulamento em assoalho com o diagnóstico de cisto nasolabial bilateral.

Submetida em abril de 2001 a exerése cirúrgica dos cistos nasais (Figura 7). 0 exame patológico mostrou que ambas formações císticas apresentavam semelhança entre si e com as estruturas do caso 1. Acompanhada por 5 meses no pós-operatório assintomática. 


\section{DISCUSSÃO}

Os cistos da região maxilar dividem-se em inflamatórios e embrionários e estes últimos subdividem-se em odontogênicos e não-odontogênicos.

Na literatura há muita discussão sobre a origem destes cistos. Em 1913, Klestadt sugeriu que estes cistos são derivados de restos ectodérmicos (epitélio das fendas faciais embriológica) na linha de fusão entre os processos nasais medial e lateral com o processo maxilar; por esta razão são chamados também de cistos fissurais ${ }^{3,5,6}$. A outra teoria para a explicar a gênese destes cistos é a de Bruggemann (1920) que seria derivado do epitélio do ducto nasolacrimal (ântero-inferior) durante sua base de desenvolvimento embrionário ${ }^{5-7}$. 0 epitélio que reveste este ducto é colunar pseudoestratificado semelhante ao que observa-se freqüentemente na parede do cisto nasolabial ${ }^{46}$. No presente estudo, todas as lesões apresentaram este revestimento epitelial. Ambas as teorias podem ser anatomicamente válidas ${ }^{4}$.

A proporção mulher:homem para os cistos bilaterais é de 5,5:1 e para os unilaterais de $3,5: 1^{8}$. Esta lesão é mais freqüente em pessoas da raça negra $3,9,10 \mathrm{com}$ maior freqüência entre a quarta e a quinta década ${ }^{7,9,11}$. Eles são usualmente unilaterais (90\%) e bilaterais em apenas $10 \%$ dos casos s $^{3,9}$.

Clinicamente, caracterizam-se pela presença de tumefação decrescimento lento na porção ventral inferior da região pinforme, anterior ao osso maxilar, sem acometê-lo e é usualmente assintomático. Em função do seu tamanho pode causar deformidade nasal e facial progressiva que consiste em protrusão do lábio superior, elevação da asa do nariz e abaulamento do sulco nasolabial assim como obstrução nasal, sendo que a dentição permanece inalterada ${ }^{6}$. Ocasionalmente pode causar dor na presença de infecção ( $50 \%$ dos casos), sendo que nestas situações podem drenar tanto no vestíbulo nasal como na cavidade oral ${ }^{6,11}$.

0 diagnóstico do cisto nasolabial é fundamentalmente clínico. A exploração mediante a palpação bidigital mostra uma tumefação flutuante entre ao assoalho do vestíbulo nasal e o sulco gengivolabial e ajuda a confirmar o diagnóstico ${ }^{6}$. Devido ser lesão de partes moles não se detecta na radiografia simples, a não ser que causem erosão óssea importante no osso maxilar. A tomografia computadorizada pode mostrar a natureza cística da lesão e sua relação com a asa nasal e o osso maxilar assim como 0 acometimento ósseo. Comumente o paciente procura serviço médico por ter notado uma alteração facial. Em alguns casos, o diagnóstico pode ocorrer fortuitamente durante um exame rotineiro otorrinolaringológico.

A lesão possui um crescimento lento e suas dimensões são variáveis $(2-3 \mathrm{~cm})^{3}$, entretanto está descrito um cisto gigante o qual drenou $250 \mathrm{ml}$ de seu conteúdo?

0 diagnóstico diferencial é realizado com outros cistos da região maxilar assim como outras massas nasais ${ }^{10}$. 0 cisto nasolabial infectado pode ser confundido com furúnculo do assoalho do vestíbulo nasal. Mas com exceção desta entidade, as características desta lesão são tão distintas que não há dúvida sobre o verdadeiro diagnóstico.
0 exame histopatológico desta lesão foi descrito pela primeira vez por Brown-Kelly em 1898 ${ }^{12}$. O cisto consiste de um epitélio respiratório (pseudoestratificado ou estratificado cilíndrico ciliado com células caliciformes - gobletcells), porém pode ocorrer metaplasia escamosa nos cistos infectados ${ }^{6}$. 0 fluido contido nos cistos é produzido pelas gobletcells.

Devido a deformidade facial progressiva, ao risco de infecção e suas seqüelas e à eventual ocorrência dolorosa no local, o paciente deve ser informado da necessidade de retirada cirúrgica. É realizada enucleação simples através de acesso intraoral, com incisão no sulco gengivolabial (Denker) e dissecção romba da lesão. Suas paredes têm íntima relação com a mucosa do assoalho do vestíbulo nasal e na maior parte das vezes acarreta uma laceração da mesma. Deve-se fazer sutura com fios absorvíveis para evitar desenvolvimento de fístula oronasal. A recorrência é rara e o prognóstico é excelente ${ }^{5,7}$. É uma patologia benigna, havendo apenas um relato por Arnold (1929) de degeneração carcinomatosa derivada do cisto nasolabial 8,13 .

\section{COMENTÁRIOSFINAIS}

Os cistos nasolabiais são muitas vezes subdiagnosticados na sua fase inicial. 0 exame físico e topográfico é o método de eleição para diagnóstico seguido de exame de imagem por tomografia computadorizada. A melhor forma de tratamento é a exerése total da lesão através de incisão intraoral sublabial na altura da fossa incisiva, que proporciona uma ampla exposição, permitindo sua completa remoção.

\section{REFERÊNCIASBIBLIOGRÁFICAS}

1. Fanibunda KB. Bilateral nasolabial cysts: a case report. Dent Pract 1970;20: 249-250.

2. Brandão GS, Ebling $H$, Souza IF. Bilateral nasolabial cyst. Oral Surg 1974;37:480-484.

3. Barzilai M. Bilateral nasoalveolar cysts: case report. Clin Radiol 1994;49(2): 140-141.

4. Waldrep AC, Capodanno JÁ. Bilateral nasolabial cysts: report of case. J Oral Surg 1966;24:347- 350.

5. Graamans K. Nasolabial cysts: diagnosis mainly based on topography?. Rhinology 1983;21: 239-249.

6. Kuriloff DB. The nasolabial cyst-nasal hamartoma. Otolaryngol Head Neck Surg 1987;96:268-272.

7. David VC, O'Connell JE. Nasolabial cyst. Clin Otolaryngol 1986;11:5-8.

8. Roed-Peterson B. Nasolabial cysts: a presentation of five patients with a review of literature. Br J Oral Surg 1969;7:84- 95.

9. Cohen MA, Hertzanu $Y$. Huge growth potential of the nasolabial cyst. Oral Surg 1985;59:441-5.

10. Karmody CS, Gallagher JC. Nasoalveolar cysts. Ann Otol 1972;81:278-283.

11. Allard RHB. Nasolabial cysts: review of the literature and report of seven cases. Int J Oral Surg 1982;11:351-359.

12. Burtschi TA, Stout RA. Bilateral nasoalveolar cysts. Oral Surg 1963;16: 271-275.

13. Egervary G, Csiba A. Bilateral nasolabial cyst. Dental Digest 1969;75:504-7.

14. Walsh-Warine GP. Nasoalveolar cysts: aetiology, presentation and treatment. J laryngol Otol 1967;81:263-273.

15. Rao RV. Nasolabial cyst. J Laryngol Otol 1955;69:352-4. 